Check for updates

Cite this: Phys. Chem. Chem. Phys., 2020, 22, 1774

Received 22nd October 2019, Accepted 11th December 2019

DOI: $10.1039 / c 9 c p 05722 f$

rsc.li/pccp

\section{Surface grafted agents with various molecular lengths and photochemically active benzophenone moieties $\dagger$}

\author{
Gaby Nordendorf, (DD ${ }^{a}$ Samuel L. Schafforz, (DD ${ }^{a}$ Eireen B. Käkel, (DD ${ }^{b}$ Shunyi Miao ${ }^{a}$ \\ and Alexander Lorenz (D) *a
}

\begin{abstract}
Homologues of benzophenone silane, a covalently graftable, photochemically active surface functionalizing agent, are investigated as surface functionalization agents for both small particles and planar substrates. In these homologues, a chlorosilane functional group and a photochemically active benzophenone oxo moiety are separated with an aliphatic spacer of varying length. The species obtained are first investigated by surface grafting on substrates (Si wafers, glass plates, and indium tin oxide coated glass plates). Si wafer samples are investigated with ellipsometry clearly indicating monolayer formation. The monolayer thickness can be controlled by the size of the aliphatic spacer group and also by the doping concentration of the solution used in the spin-casting step. The functionalized surfaces are further investigated by measuring the contact angle of a suitable organic fluid, a nematic liquid crystal. Photo exposure of these samples results in a drastically varied contact angle: The surface grafted species are still photochemically active and photo exposure leads to the addition of a nearby organic molecule (from the liquid crystalline phase) to each activated surface agent molecule. The synthesized species are then investigated as (covalently binding) surfactants in the wet planetary ball milling process aimed to fabricate solid-liquid dispersions (of Fe doped lithium niobate particles). It was found that the use of species with higher molecular length results in dispersions of small particles, functionalized with photochemically active surface agents. Indeed, they show better performance than conventional surfactants.
\end{abstract}

\section{Introduction}

Benzophenone silane, BPS, is a surface graftable crosslinker with one chlorosilane anchoring group and one photochemically active benzophenone oxo moiety. In BPS, these two functional moieties are separated by an aliphatic spacer (oxoalkyl-spacer) with a length of 3 carbon atoms. Via the chlorosilane anchoring group, BPS can easily attach to a hydroxyl-group-bearing surface in a condensation reaction. Benzophenone silane has been widely used to photochemically immobilize thin films of functional materials, ${ }^{1-21}$ in biologically relevant research, ${ }^{22-28}$ or to obtain fast switching polymer liquid crystal (LC) composites. ${ }^{29,30}$ Various types of planar substrates can be coated in a straight-forward spin casting approach by using mixtures of BPS and toluene. At the low concentrations usually applied for spin casting, one can probably

\footnotetext{
${ }^{a}$ Department of Chemistry, Paderborn University, Warburger Str. 100, 33098 Paderborn, Germany. E-mail: alexander.lorenz@uni-paderborn.de

${ }^{b}$ Institute of Chemistry, University of Kassel, Heinrich-Plett-Str. 40, 34132 Kassel, Germany

$\dagger$ Electronic supplementary information (ESI) available: NMR data for synthesized $\boldsymbol{n}$-ENE and $\boldsymbol{n}$-BPS compounds. See DOI: 10.1039/c9cp05722f
}

assume that these mixtures are perfect solutions at least at the time when they are dropped on the spinning substrates. However, the solvent evaporates during spin casting and thermal annealing.

Benzophenone is a well-known type II photoinitiator ${ }^{31-33}$ and frequently used with a tertiary amine as co-initiator. It can be applied as a photoinitiator even without the use of tertiary amines (added co-initiators), efficiently. The process of forming an active radical is a two-step process. First, benzophenone is excited. At a wavelength of $\approx 250 \mathrm{~nm}$ the excitation initially results in an exited singlet state followed by intersystem crossing to a triplet state (triplet benzophenone). At a slightly higher wavelength $\left(\approx 350 \mathrm{~nm}\right.$ ), an $\mathrm{n} \pi^{*}$ transition (with lower absorption coefficients) occurs and triplet benzophenone can be obtained, directly. In the second step, triplet benzophenone undergoes hydrogen abstraction from an aliphatic chain of a nearby molecule, for example a macromolecule, ${ }^{34}$ which results in the formation of a $\mathrm{BP}-\mathrm{H}^{\bullet}$ radical, which is usually not active towards carbon-carbon double bonds, and a second, active radical. However, the $\mathrm{BP}-\mathrm{H}^{\bullet}$ radical is also well-known to initiate the formation of a new carbon-carbon covalent bond with nearby aliphatic moieties of neighboring molecules, where 
the carboxylic oxygen is simultaneously converted to an $\mathrm{OH}$ group. Thereby, neighboring molecules can be covalently attached to the former benzophenone group. The photochemical properties of surface grafted benzophenone silanes are very much comparable to those of benzophenone. BPS can be used as a photo crosslinker in experiments where surface hydroxyl groups ${ }^{1,13,24,30,35,36}$ or surfaces with surface-near hydroxyl groups ${ }^{16}$ (such as polymer films) are first grafted with BPS and then macromolecules get photochemically attached to these benzophenone silane bearing surfaces. Alternatively, benzophenone silanes can be used as surface grafted photoinitiators ${ }^{30,37}$ to initiate radical polymerization in reactive mixtures containing suitable monomers, such as acrylates. In both cases, the activated and/or growing macroradicals can recombine with the surface bound $\mathrm{BP}-\mathrm{H}^{\bullet}$ moieties and form surface grafted macromolecules.

In the ball-milling based fabrication process of dispersions of inorganic particles such as $\mathrm{BaTiO}_{3}$ or Fe doped $\mathrm{LiNbO}_{3}$, surfactants play an important role. In order to obtain small (even in the nano-range) particles, these inorganic substances have been milled in the presence of heptane as an organic solvent and oleic acid as a surfactant. ${ }^{38-44}$ Using an organic solvent is desirable to make use of the dispersions obtained and dope target samples such as LCs, polymers or other samples with small, surface functionalized particles. An organic solvent can be removed from these samples easily (without varying the chemical composition of the doped substances). It has become clear that oleic acid can in some cases bind covalently $^{42}$ to the particle surfaces during the milling process and result in small particles functionalized with a stable organic monolayer, which might be very important to obtain stable dispersions or nano-dispersions with the desired stability. The ball milling process is delicate and depends for example on the milling time, the type of balls used, and the milling energy. Anyway, alternative surface functionalizing agents should be investigated.

In this work, homologues (with an alkyloxo spacer length of $n=4-12$ carbon atoms) of BPS were synthesized (the synthesis route is summarized in Scheme 1) by following the two-stepped procedure published for $\mathrm{BPS}^{24}$ and replacing 3-bromopropen with higher homologues of terminal bromoalkenes. In the first step, the intermediate products (n-ENE intermediates) were obtained and then converted to the final BPS homologues (n-BPS).

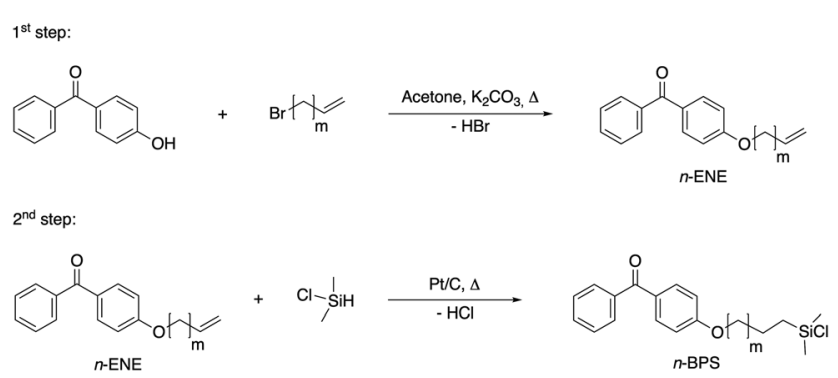

Scheme 1 Reactions for the two-step synthesis of the intermediate products $n$-ENE and the final products $n$-BPS (with $n=m+2$ carbon atoms).
For the surface functionalization of substrates, the BPS homologues obtained were dissolved in toluene. These solutions were spin cast on silicon wafers. The spin coated samples were thermally annealed and subsequently cleansed with an organic solvent. The surface functionalized, cleansed samples were investigated with ellipsometry to reveal thickness variations of the monolayers of BPS homologues. The results were very interesting and partially unexpected. The variations were caused by the varying spacer length of the BPS homologues and also by concentration variations of the solution used in the spin casting step. Contact angle variations were observed upon photo exposure: The species were indeed still photochemically active. The species were added as surface agents in the wet planetary ball milling process aimed to fabricate solid-liquid dispersions (of iron doped lithium niobate, Fe: $\mathrm{LiNbO}_{3}$ ) in heptane. It was found that species with higher molecular length were indeed suitable to yield the desired dispersions of particles bearing a photochemically active surface functionalization.

\section{Results and discussion}

The experimental results are presented in the following subsections along with a brief discussion of the experimental outcome.

\subsection{Synthesis results}

BPS and its homologues (with spacer length between 4 and 12 carbon atoms) were successfully synthesized. The synthesis procedure turned out to be feasible for all investigated homologues, as expected. Anyway, the yields obtained varied. The yield for the intermediate $\boldsymbol{n}$-ENE products (Table 1) was high (78\%) for 3-ENE, and low for 4-ENE (34\%) and 5-ENE (25\%). The synthesis of 6- and 7-ENE led to yields higher than $65 \%$ and the yield then steadily decreased with the increasing length of the aliphatic chain of the terminal bromoalkene used.

In contrast, the hydrosilation step always resulted in high yields (Table 2): the average yield (not considering the outlier value of 10-BPS) was $76 \%$ with a standard deviation of $11 \%$. The chain length of the aliphatic spacer had no influence on the conversion seen.

Table 1 Summary of physical data and conversion for the synthesized intermediate $\boldsymbol{n}$-ENE products

\begin{tabular}{llllll}
\hline Compound & $\begin{array}{l}\text { Molar mass } \\
{\left[\mathrm{g} \mathrm{mol}^{-1}\right]}\end{array}$ & $\begin{array}{l}\text { Melting } \\
\text { point }\left[{ }^{\circ} \mathrm{C}\right]\end{array}$ & Color & $\begin{array}{l}\text { Mass } \\
{[\mathrm{g}]}\end{array}$ & $\begin{array}{l}\text { Conversion } \\
{[\%]}\end{array}$ \\
\hline 3-ENE & 238 & 76.9 & White & 3.65 & 78 \\
4-ENE & 252 & Liquid & Clear & 1.70 & 34 \\
5-ENE & 266 & 37.3 & White & 0.66 & 25 \\
6-ENE & 280 & Liquid & Clear & 3.78 & 65 \\
7-ENE & 294 & Liquid & Clear & 4.78 & 81 \\
8-ENE & 308 & 34.4 & White & 3.15 & 51 \\
9-ENE & 322 & 35.3 & White & 2.63 & 41 \\
10-ENE & 336 & 44.7 & White & 2.81 & 42 \\
11-ENE & 350 & 47.8 & White & 2.57 & 37 \\
12-ENE & 364 & 53.5 & White & 0.35 & 25
\end{tabular}


Table 2 Summary of physical data and conversion for the synthesized n-BPS compounds

\begin{tabular}{llllll}
\hline Compound & $\begin{array}{l}\text { Molar mass } \\
{\left[\mathrm{g} \mathrm{mol}^{-1}\right]}\end{array}$ & $\begin{array}{l}\text { Melting } \\
\text { point }\left[{ }^{\circ} \mathrm{C}\right]\end{array}$ & $\begin{array}{l}\text { Mass } \\
{[\mathrm{g}]}\end{array}$ & $\begin{array}{l}\text { Conversion } \\
{[\%]}\end{array}$ \\
\hline 3-BPS & 332.6 & $\approx 33$ & White & 3.31 & 67 \\
4-BPS & 346.6 & Liquid & White & 0.88 & 76 \\
5-BPS & 360.6 & 53 & White & 0.45 & 80 \\
6-BPS & 374.6 & Liquid & Brown & 3.49 & 79 \\
7-BPS & 388.6 & 42 & Brown & 1.29 & 65 \\
8-BPS & 402.6 & Liquid & Brown & 3.35 & 93 \\
9-BPS & 416.6 & 57.5 & White & 2.73 & 87 \\
10-BPS & 430.6 & 42 & White & 0.70 & 20 \\
11-BPS & 444.6 & 53 & White & 2.00 & 61 \\
12-BPS & 458.6 & 46 & White & 0.31 & 74
\end{tabular}

\subsection{Monolayer thickness}

Initially, the length of $\boldsymbol{n}$-BPS-O-SiH $\mathrm{S}_{3}$ molecules in their all-trans conformation was measured in a ball-and-stick model in an optimized molecular geometry (UFF force field) in Avogadro. ${ }^{45}$ In this straight-forward approach, the Cl-atom of $\boldsymbol{n}$-BPS was replaced by a $\mathrm{O}-\mathrm{SiH}_{3}$ moiety, which probably was a preferable approximation for a surface-bound molecule (preferable to a Cl-ligand).

The furthest distance between the Si-atom (silane group) and one selected hydrogen atom of the benzophenone group was measured as shown in Fig. 1 for 11-BPS. The obtained values for all investigated BPS homologues are listed in Table 3. The length obtained represents the maximum possible thickness of a $\boldsymbol{n}$-BPS monolayer on a substrate surface in the case of perpendicular (brush-like) molecular alignment (when neglecting the covalent silicon-oxygen bonds connecting the Si-atom covalently to the substrate). In the approach presented, the part of the molecules actually contributing to the measurable layer thickness is accounted for. This thickness obtained is a feasible value, because the covalently bound surface-oxygen is part of a native $\mathrm{SiO}_{2}$ layer present at the substrate surfaces.

The film thickness derived from ellipsometric measurements (colored symbols) along with the estimated maximum values for monolayers of surface grafted molecules (gray shaded squares) is shown in Fig. 2. A linear trend line can be
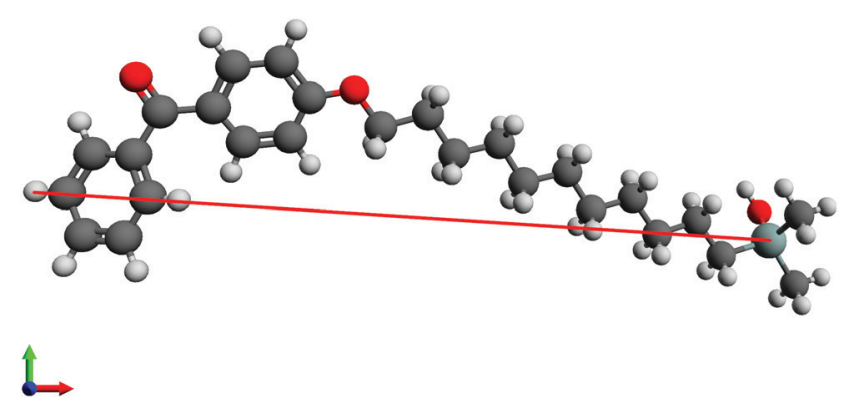

Fig. 1 The ball-and-stick model of 11-BPS in all-trans conformation in an optimized geometry (Avogadro, UFF force field) is shown exemplarily. The red colored line indicates the molecular length measured ( $23.75 \AA$ ). Here, showing the crystal structure of the $\mathrm{SiO}_{2}$ surface (to which the BPS molecule is attached in a real sample) would reduce the clarity, and hence a covalently bound BPS-O- $\mathrm{SiH}_{3}$ is shown instead.
Table 3 Estimated molecular length of surface grafted $\boldsymbol{n}$-BPS molecules (all-trans conformation) contributing to the film thickness, where $n$ represents the number of carbon atoms in the alkyl chain

\begin{tabular}{ll}
\hline Compound & Molecular length [A] \\
\hline 3-BPS & 14.48 \\
4-BPS & 15.06 \\
5-BPS & 16.73 \\
6-BPS & 17.40 \\
7-BPS & 19.02 \\
8-BPS & 19.81 \\
9-BPS & 21.14 \\
10-BPS & 22.23 \\
11-BPS & 23.75 \\
12-BPS & 24.66
\end{tabular}

used to describe the estimated molecular length in the all-trans conformation, as indicated in the figure. The slope of this trend line was $\approx 0.12 \mathrm{~nm}$ (slightly lower than a typical $\mathrm{C}-\mathrm{C}$ bond length of $0.154 \mathrm{~nm}$ ) per carbon atom $n$. For all samples, the experimentally measured film thickness was below the estimated maximal monolayer thickness, which clearly indicated the formation of monolayers, where the molecular conformation and/or orientation differed (with a few exceptions) from the all-trans conformation with perpendicular molecular alignment. In the samples obtained from solutions with an intermediate concentration, the film thickness initially (for a spacer length of 3,5 or 6 carbon atoms) decreased with increasing spacer length. Looking at the higher ( 5 to 11 carbon atoms) spacer length, one can also see that the concentration now increased linearly with increasing spacer length. Again, the data were fitted with a linear trend line. The slope obtained was $\approx 0.11 \mathrm{~nm}$ per carbon atom $n$, and thus comparable to the slope seen in the estimated molecular length in the all-trans confirmation. However, the layer thickness was much $(0.8 \mathrm{~nm})$ smaller than would be expected for monolayers with all-trans conformation and perpendicular anchoring. Using a low concentration (Fig. 2, yellow diamonds) led to even smaller layer thickness and a smaller slope ( $0.055 \mathrm{~nm}$ per carbon atom) of the trend line. The layer thickness measured for the high

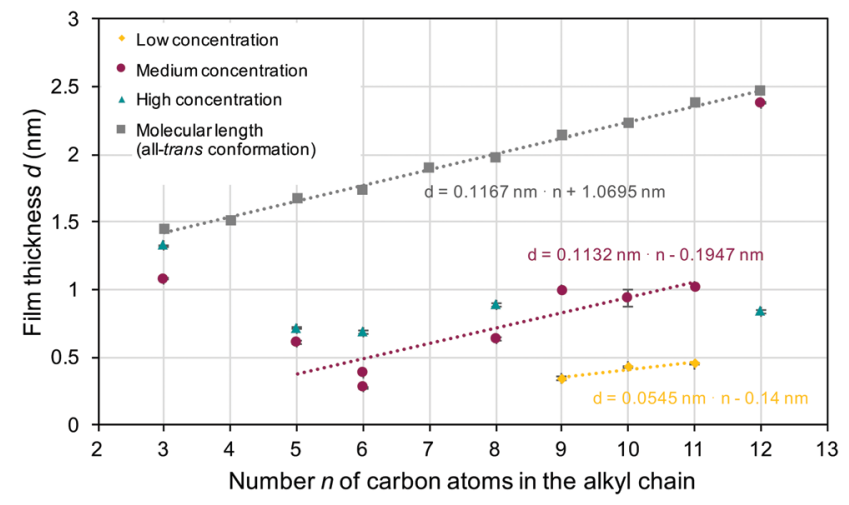

Fig. 2 Film thickness derived by spectroscopic ellipsometry for surface grafted $\boldsymbol{n}$-BPS monolayers obtained from coating solutions with various $\boldsymbol{n}$-BPS concentrations. In addition, the estimated molecular length of the homologues in the all-trans conformation as the limiting thickness of a monolayer is shown. 
concentration (taupe colored triangles) resulted in an almost constant value of $\approx 0.7 \mathrm{~nm}$. A monolayer with perpendicular orientation of the surface grafted molecules was selectively found in 3-BPS (independent of the concentration) and 12-BPS (at a medium concentration). Monolayers were successfully formed in all samples prepared. The impact of the doping concentration on the monolayer thickness was, however, surprising. The samples were spin coated from a liquid phase, which possessed various $\boldsymbol{n}$-BPS concentrations. In general, a high initial concentration led to a constant monolayer thickness. In the samples made with lower concentrations of the liquid phase, the monolayer thickness (determined by the molecular conformation of the grafted molecules and the grafting density) was varied. An alternative approach to coat surfaces with BPS is to immerse samples in a BPS solution, ${ }^{1,6,13}$ which can be heated or slowly evaporated during the grafting step without creating convection fluxes as during spin casting. If so, the grafting density can be even higher than in spin cast samples and the resulting layer thickness of monolayers can be affected. In the spin casting approach, two opposing processes took place simultaneously: The $\boldsymbol{n}$-BPS concentration in the bulk of the liquid phase was lowered by the condensation reaction with surface $\mathrm{OH}$-groups (reaction at the solid-liquid interface), while it was increased by evaporation of the solvent. In the spin casting approach, the concentration of $\boldsymbol{n}$-BPS in the bulk of the liquid phase was simultaneously increased, since the solvent evaporated.

A non-polar solvent was used. Therefore, one can speculate that the dissolved $\boldsymbol{n}$-BPS molecules (with polar end-groups) should possess a surface excess and were thus pushed to the surfaces upon increasing the overall concentration in the liquid phase. Maybe even lyotropic phases can be formed at higher (increasing with time) concentrations of $\boldsymbol{n}$-BPS. Here, the grafting from such a lyotropic phase can be described with two processes: One possibility, which can lead to characteristic grafting kinetics, is that the concentration of the freely dissolved species is kept constant in the liquid phase, if a lyotropic phase (such as a micellar phase) was formed. Should the concentration decrease due to the grafting process, species will re-dissolve from the micelles present. Alternatively, the monolayer could be grafted through a process, where first micelles were adsorbed on the surfaces. It would be desirable to study the phase behavior of $\boldsymbol{n}$-BPS species at various concentrations in scattering experiments. Such experiments turned out to be challenging in a preliminary approach, which might be caused by the non-typical structure of $\boldsymbol{n}$-BPS with two (polar) functional groups, separated by an aliphatic spacer. Anyway, the monolayer thickness seen in the higher homologues $(n>9)$ (where formation of lyotropic phases seems more likely) was less concentration dependent, than in the smaller molecules. Understanding the phase behavior of $\boldsymbol{n}$-BPS in more detail can potentially help to develop a detailed understanding of the grafting process of these versatile, surface graftable, photochemically active molecules.

\subsection{Contact angle of $\mathrm{E} 7$}

The contact angle of a suitable organic fluid, the nematic liquid crystal E7, on the treated substrates (references and spin coated
Table 4 Contact angles of E7 on the surface of reference samples

\begin{tabular}{lllc}
\hline Substrate & Plasma treatment & UV exposure & Contact angle $\left[{ }^{\circ}\right]$ \\
\hline Si wafer & - & - & $6.5 \pm 1.3$ \\
& Post & - & $2.1 \pm 0.3$ \\
& - & Post & $10.3 \pm 0.5$ \\
Glass & Post & - & $5.8 \pm 0.2$ \\
& - & - & $8.9 \pm 0.9$ \\
& Post & Post & $5.0 \pm 0.0$ \\
& Post & Post & $3.5 \pm 0.0$ \\
ITO coated glass & - & - & $10.4 \pm 1.3$ \\
& Post & - & $2.7 \pm 0.6$ \\
& - & Post & $11.2 \pm 1.0$ \\
& Post & Post & $4.6 \pm 1.7$ \\
\end{tabular}

samples) was investigated. The results obtained in the reference samples are summarized in Table 4 . The cleansed surfaces were activated first by plasma treatment and then by UV exposure. In the non-treated substrates, small contact angles of $6.5^{\circ}$ (Si-wafers), $8.9^{\circ}$ (glass plates), and $10.4^{\circ}$ (ITO glass plates) were found. After plasma treatment, these angles were varied to smaller values of $2.1^{\circ}$ (Si-wafers), $5^{\circ}$ (glass plates), and $2.7^{\circ}$ (ITO glass plates), which can be explained by the increased surface polarity (activated surfaces, presence of surface $\mathrm{OH}$ groups). UV treatment of cleaned samples resulted in contact angles of $10.3^{\circ}$ (Si-wafers), $11^{\circ}$ (glass plates), and $11.2^{\circ}$ (ITO glass plates). These changes were small. Anyway, UV exposure of previously plasma treated samples led to a slight increase to $5.8^{\circ}$ (Si-wafers), $3.5^{\circ}$ (glass plates), and $4.6^{\circ}$ (ITO glass plates), which were intermediate values smaller than in the bare samples but higher than in the plasma treated samples. UV exposure in the bare samples or in the presence of E7 led to a slight deactivation of the surfaces as compared to the plasma treated samples.

In surface grafted samples (9-BPS) (Table 5), which were plasma treated prior to the grafting step, the contact angles were drastically increased to $\approx 20^{\circ}$ (Si-wafers) and $>13^{\circ}$ (glass plates). For ITO coated glasses, such an increase was selectively seen, if methyl cyclohexane was used as a solvent. If the surface grafted samples were exposed to UV light, the contact angle decreased, as expected, since the surfaces were now

Table 5 Contact angles of E7 on 9-BPS grafted surfaces prior to and after UV exposure (now leading to photochemical modification of the surfaces)

\begin{tabular}{llll}
\hline Substrate & Solvent & UV exposure & Contact angle $\left[^{\circ}\right]$ \\
\hline Si wafer & 2-Butanone & - & $22.6 \pm 2.5$ \\
& & Post & $14.0 \pm 0.8$ \\
& Methyl cyclohexane & - & $19.9 \pm 0.8$ \\
Glass & Post & $19.5 \pm 0.4$ \\
& 2-Butanone & - & $13.0 \pm 2.7$ \\
& & Post & $12.1 \pm 0.9$ \\
& & - & $19.1 \pm 2.7$ \\
ITO coated glass & 2-Butanone & - & $20.4 \pm 0.8$ \\
& & Post & $5.0 \pm 0.0$ \\
& \multirow{2}{*}{ Methyl cyclohexane } & - & $15.7 \pm 1.1$ \\
& & Post & $10.6 \pm 1.7$
\end{tabular}



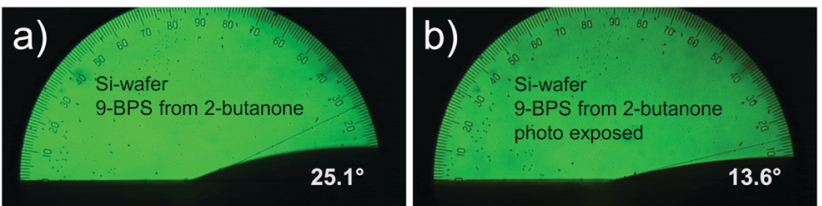

Fig. 3 Contact angle of E7 investigated on Si wafer samples. (a) Coated with 9-BPS and (b) after UV exposure.

functionalized with photochemically attached LC molecules. This decrease (from $22.6^{\circ}$ to $14^{\circ} ; \Delta=8.6^{\circ}$ ) was most pronounced in Si-wafer samples grafted by using 2-butanone 9-BPS solutions and ITO glasses treated with methyl cyclohexane (from $15.6^{\circ}$ to $10.6^{\circ} ; \Delta=5^{\circ}$ ). Example images recorded with a suitable camera attached to the contact angle meter are shown (Fig. 3). The effect was less pronounced (i.e. $<2^{\circ}$ ) in the alternative samples. One can speculate that the UV intensity at the surfaces was maybe too small (exposure through the LC layer) in the latter experiments.

\subsection{DLS on functionalized particles}

The light scattering data obtained in samples milled with added 7-BPS are shown in Fig. 4. Useful data could already be obtained after $16 \mathrm{~h}$ of grinding time. The curves shown had a polydispersity index $<0.3$ and showed a Gaussian-like distribution of particle sizes. At a grinding time of $18 \mathrm{~h}$, the average particle diameter was $\approx 500 \mathrm{~nm}$. The particles obtained were comparably well dispersed. For reference, also lecithin and oleic acid were used as surfactants. Milling in the presence of 7-BPS resulted in samples which could be easily investigated with DLS, whereas the reference samples were still contaminated with larger particles which corrupted the measurements. No data could be extracted from these measurements. It is probably possible to find small particles in such samples after elimination of large particles by filtration or centrifugation. However, the use of $\boldsymbol{n}$-BPS homologues was preferable, since the well-known alternative surfactants cannot yield photochemically active particles.

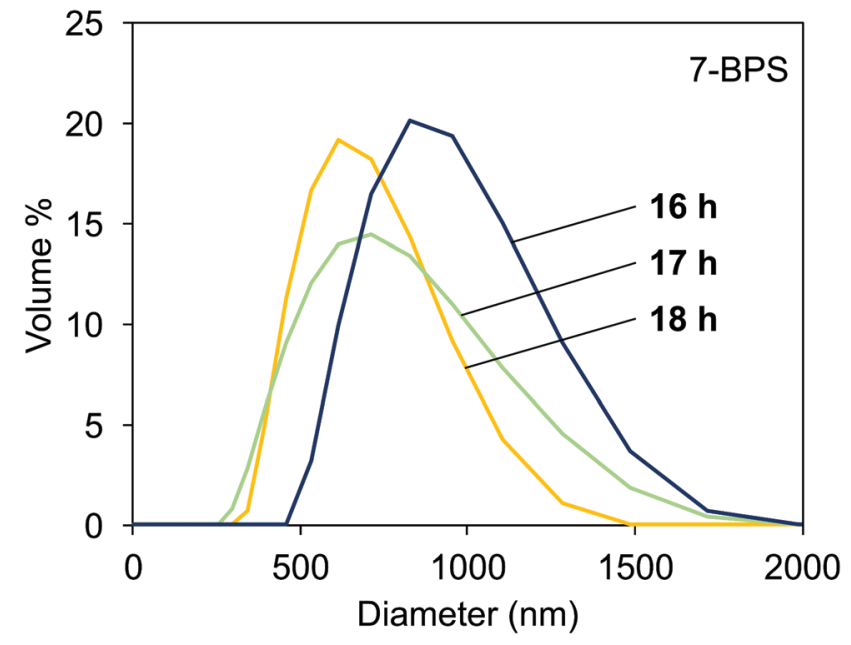

Fig. 4 Particle distribution measured with DLS in dispersions of milled $\mathrm{Fe}: \mathrm{LiNbO}_{3}$ particles, milled in the presence of 7-BPS for 16, 17, and $18 \mathrm{~h}$.

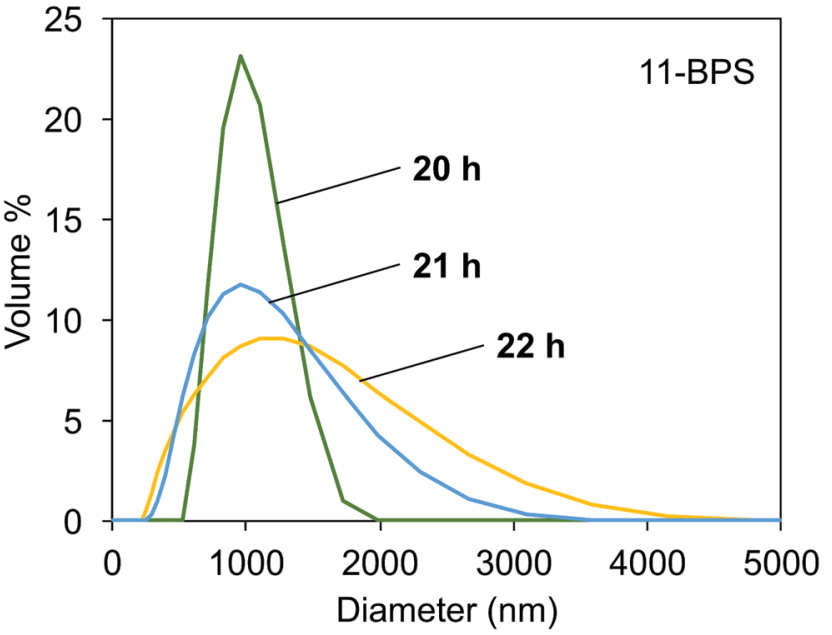

Fig. 5 Particle distribution measured with DLS in dispersions of milled $\mathrm{Fe}: \mathrm{LiNbO}_{3}$ particles, milled in the presence of 11-BPS for 20, 21, and $22 \mathrm{~h}$.

The DLS data measured in samples milled in the presence of 11-BPS after 20, 21, and $22 \mathrm{~h}$ of milling time had passed are shown in Fig. 5. The polydispersity index measured again was $<0.3$, which showed that samples were not monodisperse but DLS was still a suitable method to study their properties. After $20 \mathrm{~h}$ of milling time had passed, the particle size distribution curve was relatively narrow and had a maximum at $\approx 1000 \mathrm{~nm}$. The particle size distribution became wider at higher milling times. High milling times were required and the samples initially contained two types of particles, smaller and larger particles. The contribution of large particles is not seen in the size distribution at $20 \mathrm{~h}$, because these particles were still large enough to segregate at the bottom plane of the DLS cuvettes, where they could not contribute to the scattering. Due to further progress of the milling process, the particle size distribution became wider as seen in the curves recorded at $21 \mathrm{~h}$ and $22 \mathrm{~h}$ milling time. Now, also smaller particles were dispersed than in the samples taken at a milling time of $20 \mathrm{~h}$. In addition, these dispersions also contained even larger particles than were seen at $20 \mathrm{~h}$ milling time. These particles could be formed by milling progress of larger particles still present in the grinding jars, but (as explained before) not contributing to the scattering in the DLS experiments conducted in samples taken at a milling time of $20 \mathrm{~h}$. It is also possible that the large particle sizes seen in the scattering curves in samples at $21 \mathrm{~h}$ and $22 \mathrm{~h}$ were due to aggregation of smaller particles. The particle size distributions shown are promising, since they show how the milling progress resulted in dispersions of small, surface functionalized inorganic particles, which may find applications in doped polymer films and as dopants for soft phases, such as liquid crystals and lyotropic phases.

The milling experiments clearly showed that the presence of 7-BPS and 11-BPS led to the successful generation of dispersions of relatively small, milled particles. The milling process even worked more efficiently than in reference experiments with oleic acid and lecithin. The particles obtained in the presence of $\boldsymbol{n}$-BPS can be expected to possess a shell, probably 
even a monolayer shell, of $\boldsymbol{n}$-BPS attached to their polar $\mathrm{Fe}: \mathrm{LiNbO}_{3}$ surface.

The surface free energy of particles can be reduced by the presence of a solvent (through wetting of the particle's surfaces) and (more efficiently) by the presence of a surfactant. The use of surfactants in the wet high energy planetary ball milling process (surfactant assisted high energy ball milling) has been comprehensively reviewed. ${ }^{46}$ A surfactant (for example the polar head group) can be physically adsorbed on the particle's surfaces and stabilize the internal interface of the solid-liquid dispersion through London-dispersion forces, residing between the aliphatic tail of the surfactant molecule and solvent molecules. The presence of a surface-active substance can prevent particle agglomeration or cold welding processes. Moreover, high energy ball milling is sometimes discussed as a means of mechanochemical synthesis: ${ }^{42,46,47}$ Oleic acid can in general be used as a surfactant. However, in milled dispersions of barium titanate particles it was found that oleic acid was transformed to a metal oleate compound (with ionic bonds between the particle surface and the surface covering layer). The presence of a surface grafted layer can stabilize a dispersion more efficiently than a physically adsorbed interfacial agent. The role of $\boldsymbol{n}$-BPS is schematically shown and compared to milling with just a solvent in Fig. 6.

The $\boldsymbol{n}$-BPS coated particles obtained are very promising to be incorporated in bulk materials such as polymer films (where the benzophenone group can attach to the polymer via the photo-induced formation of C-C-bonds, or particles with an

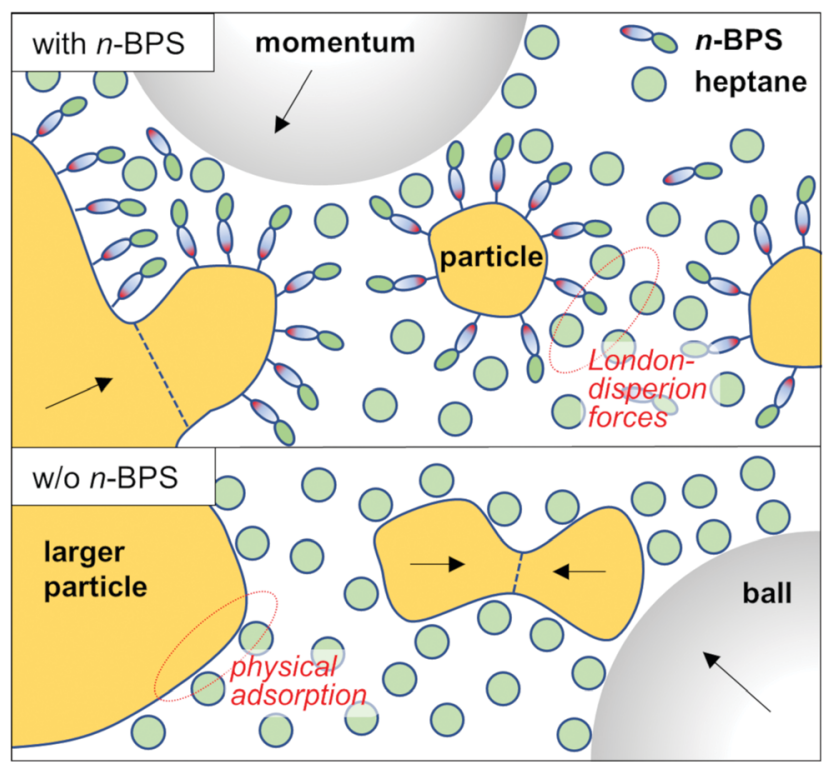

Fig. 6 Schematic of the milling process in the presence of $\boldsymbol{n}$-BPS and without $\boldsymbol{n}$-BPS. In the first case, the surfaces of oxide particles are grafted with a monolayer of $\boldsymbol{n}$-BPS. The surface free energy is thereby reduced. The solid-liquid dispersion is stabilized through London-dispersion forces (between the grafted molecules and the solvent). Without $\boldsymbol{n}$-BPS, the surface free energy can also be lowered but now, selectively, by physisorption of solvent molecules. If so, agglomeration and even fusion of smaller particles cannot be efficiently prevented and the particles can easily segregate: The solid-liquid dispersion is not well stabilized. attached photoinitiator). Such particles with an attached photoinitiator can be applied in grafting from experiments, where surface attached oligomers or polymers can be grown in from the particle surfaces. Here, the particles should be dispersed in a solution containing suitable monomers (such as acrylate monomers). Exposure to light will initiate a radical polymerization reaction where the growing chains are attached to the particle surfaces.

\section{Experimental section}

\subsection{Synthesis}

The two-stepped synthesis route for BPS has been well described previously. ${ }^{6,13}$ The description of the synthesis of one higher homologue (6-BPS) is given in the following for completeness. Comments on the appearance, melting point data (as seen in melting point tubes), the obtained masses, and conversion for both synthesis steps are summarized in Table 1 (intermediate products) and Table 2 (final products). The homologous series was synthesized by replacing allylbromide in the first synthesis step of BPS ${ }^{6,13}$ with linear terminal bromo-alkenes (homologues of allylbromide). Therefore, a higher homologue of the intermediate product was obtained in the Williamson ether synthesis step, which was subsequently hydrosilated (second step) with chlorodimethylsilane, to finally obtain BPS-homologues with oxoalkyl spacer length in the range of 4-12 carbon atoms ( $\boldsymbol{n}$-BPS). As an example, the synthesis of (4-(hex-5-en-1-yloxy)phenyl)(phenyl)methanone (6-ENE) and (4-((6-(chlorodimethylsilyl)hexyl)oxy)phenyl)(phenyl)methanone (6-BPS) is described below. The hydrosilation step was conducted in a dry argon atmosphere. All synthesis- and post-synthesis purification steps were carried out under yellow light conditions (in a yellow-lit lab and fume hood) since the benzophenone moiety was sensitive to UV-C radiation.

Williamson ether synthesis of (4-(hex-5-en-1-yloxy)phenyl)(phenyl)methanone (6-ENE). Typically, 4-hydroxybenzophenone $(24.77 \mathrm{mmol})$ was dissolved in $20 \mathrm{~mL}$ of acetone in a three necked round bottom flask and the mixture was stirred (magnetic stirrer). The starting terminal-bromo-alkene compound $(0.020 \mathrm{~mol})$, here 6-bromo-1-hexene (20.01 $\mathrm{mmol})$, was added and dissolved. After adding one equivalent of $\mathrm{K}_{2} \mathrm{CO}_{3}(24.69 \mathrm{mmol})$, the mixture was stirred and heated to reflux for $5 \mathrm{~h}$. The mixture was subsequently allowed to cool to room temperature and $20 \mathrm{~mL}$ of deionized water was added. The resulting solution was extracted four times with $20 \mathrm{~mL}$ of diethyl ether and the combined ether phases were washed four times with $20 \mathrm{~mL}$ of aqueous $\mathrm{NaOH}$ solution (10\%). The organic phase was dried with $\mathrm{Na}_{2} \mathrm{SO}_{4}$ overnight and filtered off and collected in a round bottom flask. The filter residue was washed with dried diethyl ether. The solvent was removed under reduced pressure and the raw product was obtained. For further purification, the raw product was recrystallized (crystallizes in the fridge but is liquid at RT) from methanol to yield $3.78 \mathrm{~g}(65 \%)$. The synthesis of the other homologues resulted in higher yields in the range of 25-81\%, which are summarized in Table 1. 
${ }^{1} \mathrm{H}$ NMR (500 MHz, $\mathrm{CDCl}_{3}, \delta$ in ppm): $1.60\left(\mathrm{~m}, 2 \mathrm{H}, \mathrm{CH}_{2}-\mathrm{CH}_{2}\right)$, $1.92\left(\mathrm{~m}, 2 \mathrm{H}, \mathrm{CH}_{2}-\mathrm{CH}_{2}\right), 2.15\left(\mathrm{~m}, 2 \mathrm{H}, \mathrm{CH}_{2}-\mathrm{CH}_{2}\right), 4.05(\mathrm{~m}, 2 \mathrm{H}$, $\left.\mathrm{O}-\mathrm{CH}_{2}\right), 4.98\left(\mathrm{~m}, 1 \mathrm{H}, \mathrm{CH}_{2}=\mathrm{CH}_{2}\right), 5.05\left(\mathrm{~m}, 1 \mathrm{H}, \mathrm{CH}_{2}=\mathrm{CH}_{2}\right), 5.83$ (m, $1 \mathrm{H}, \mathrm{CH}_{2}=\mathrm{CH}_{2}$ ), 6.95-7.82 (various $\mathrm{m}, 9 \mathrm{H}, \mathrm{C}-\mathrm{H}_{\text {arom. }}$ ).

The NMR data of the other homologues are included in the NMR section of the ESI. $\dagger$

Synthesis of (4-((6-(chlorodimethylsilyl)hexyl)oxy)phenyl)(phenyl)methanone (6-BPS). The intermediate product (6-ENE) (3.28 g, $11.74 \mathrm{mmol})$ and $\mathrm{Pt} / \mathrm{C}$ (10 wt\%, catalytic amount) were placed in a three necked round bottom flask equipped with a Dimroth type condenser under a dry argon atmosphere. Dimethylchlorosilane $(6 \mathrm{~mL}, 54.03 \mathrm{mmol})$ was slowly injected through a septum. The reaction mixture was heated to reflux for $5 \mathrm{~h}$ and subsequently cooled down using an ice bath. Excess dimethylchlorosilane was removed under reduced pressure. The residue was dissolved in dry diethyl ether and the catalyst was removed by filtration (CHROMAFIL PET-20/25). The product $(3.49 \mathrm{~g}, 79 \%)$ was obtained after evaporation of the ether. Because of the sensitivity of the product, it was not further purified.

${ }^{1} \mathrm{H}$ NMR (500 $\mathrm{MHz}, \mathrm{CDCl}_{3}, \delta$ in ppm): 0.01-0.09 and 0.41 $\left(\mathrm{m}, 6 \mathrm{H}, \mathrm{Si}-\mathrm{CH}_{3}\right), 0.53\left(\mathrm{~m}, 2 \mathrm{H}, \mathrm{Si}-\mathrm{CH}_{2}\right), 1.30-1.52\left(\mathrm{~m}, 6 \mathrm{H}, \mathrm{CH}_{2}-\right.$ $\left.\mathrm{CH}_{2}-\mathrm{CH}_{2}\right), 1.82\left(\mathrm{~m}, 2 \mathrm{H}, \mathrm{CH}_{2}-\mathrm{CH}_{2}-\mathrm{CH}_{2}\right), 4.04\left(\mathrm{~m}, 2 \mathrm{H}, \mathrm{O}-\mathrm{CH}_{2}\right)$, 6.94-7.81 (various $\mathrm{m}, 9 \mathrm{H}, \mathrm{C}-\mathrm{H}_{\text {arom.) }}$.

The measured NMR data of the other homologues are included in the ESI. $\uparrow$ These data showed that the final product was (at least in the $\mathrm{CDCl}_{3}$ solutions contained in the NMR tubes) partially converted: the chlorosilane functional group was converted to a silanol functional group (in the presence of residual water). Anyway, the results obtained in the following investigation steps clearly showed that the surface grafting step (condensation reaction with surface $\mathrm{OH}$-groups) worked well and surface grafted monolayers were obtained from all homologues investigated.

\subsection{Methods}

Surface immobilization of BPS homologues. The silicon substrates were cleaned by sonication $(2 \mathrm{~min})$ sequentially in each of the following solvents: deionized water, acetone and iso-propyl alcohol. Prior to cleaning a substrate with the next solvent in the sequence, the pre-cleansed substrate was dried under nitrogen gas flow. Following the wet cleaning process, the substrates were treated with oxygen plasma (Diener, $420 \mathrm{~W}$, 0.7 bar, $25^{\circ} \mathrm{C}$ ) for $5 \mathrm{~min}$. To ensure the surfaces were activated, before the spin coating process, each sample additionally received UV-treatment $(20 \mathrm{~min}$ ) directly before it was placed on the vacuum chuck of the spin coater. Each substrate was spin cast with $20 \mu \mathrm{L}$ of a mixture (solution, dispersion) of a BPS homologue $(0.5 \mathrm{mg})$ in toluene $(1.5 \mathrm{~mL})$ (Uvasol) for $2: 15 \mathrm{~min}$ at 50 rps. Subsequently, the spin cast substrates were placed on a temperature controlled hot plate $\left(55^{\circ} \mathrm{C}\right)$ for $40 \mathrm{~min}$ in a fume hood to promote occurrence of the condensation reaction (surface attachment) and to remove the solvent. The substrates were allowed to cool down to room temperature again and any excess (non-grafted) BPS homologue was removed by rinsing with $350 \mu \mathrm{L}$ of toluene four times. After every second rinse, the sample was dried (blowing ball) with air.
Table 6 Concentration of BPS solutions used for spin casting

\begin{tabular}{llll}
\hline & $\begin{array}{l}\text { Low } \\
\text { concentration } \\
{\left[\mathrm{mol} \mathrm{L}^{-1}\right]}\end{array}$ & $\begin{array}{l}\text { Medium } \\
\text { concentration } \\
{\left[\mathrm{mol} \mathrm{L}^{-1}\right]}\end{array}$ & $\begin{array}{l}\text { High } \\
\text { concentration } \\
{\left[\mathrm{mol} \mathrm{L}^{-1}\right]}\end{array}$ \\
\hline 3-BPS & & 0.00120 & 0.00240 \\
5-BPS & & 0.00129 & 0.00258 \\
6-BPS & & 0.00125 & 0.00250 \\
8-BPS & 0.00064 & 0.00166 & 0.00332 \\
9-BPS & 0.00062 & 0.00128 & \\
10-BPS & 0.00075 & 0.00124 & \\
11-BPS & & 0.00150 & 0.00232 \\
12-BPS & & 0.00116 &
\end{tabular}

The concentration of the coating solutions was adjusted and three series of concentrations were used, as shown in Table 6.

Ellipsometry. The samples with surface immobilized $\boldsymbol{n}$-BPS layers were investigated with spectroscopic ellipsometry (Woollam, Variable Angle Spectroscopic Ellipsometer, auto retarder set to high accuracy mode to ensure the recorded phase data were accurate) in the visible wavelength range $(400-800 \mathrm{~nm})$, with a step width of $2 \mathrm{~nm}$. Amplitude and phase were measured for each data point and three angles of incidence $65^{\circ}, 70^{\circ}$ and $75^{\circ}$ were investigated. Each cleansed sample was investigated after plasma treatment and prior to the spin casting step to measure the thickness of the native $\mathrm{SiO}_{2}$ layer present at the silicon surface. Analysis of the ellipsometric data was conducted with the WVASE ${ }^{\circledR}$ software (J. A. Woollam Co.). Based on a model structure comprised of the different layers of the sample, the thickness of the BPS layer was obtained by assuming a Cauchy material as fitting parameter with $A_{n}=1.409$ and $B_{n}=0.01$. The $\mathrm{Si}$ and $\mathrm{SiO}_{2}$ layers were fitted based on well-known models ${ }^{48}$ and the thickness measured in the samples prior to BPS immobilization was then used for modeling the data measured for the $\boldsymbol{n}$-BPS covered substrates. For completeness, the influence of the surface roughness and thickness non-uniformity was investigated and it was found that they typically did not affect the results.

Contact angle measurements. The contact angle of the nematic liquid crystal E7 on different substrate surfaces (glass, ITO coated glass, and silicon wafer), modified through surface treatment, was investigated using a contact angle meter (Erma Inc., No. 20593, fitted with a Schott KL 150B cold light source). All substrates were initially cleaned using the aforementioned wet cleaning procedure. The bare substrates were additionally cleaned by plasma treatment (Harrick Plasma Cleaner/Sterilizer PDC-32G). As a reference, for each type of substrate, the contact angle of E7 (deposited droplets) was measured prior to and after plasma treatment. The different types of substrates were spin coated (followed by annealing and rinsing) with solutions of 9-BPS and 4-BPS in the two different (dry) solvents 2-butanone and methyl cyclohexane $(0.5 \mathrm{mg}$ of BPS in $1.5 \mathrm{~mL}$ of solvent). First, the contact angle of the LC E7 was measured. Subsequently, E7 was manually spread on each substrate, in order to cover it with a continuous E7 film. The substrates were then exposed to UV light (10 $\mathrm{min}$ ) to initiate photo-reaction (of the benzophenone-functional groups and LC molecules). Since Si-wafer samples were not transparent to UV light, all substrates 
were exposed top-down through the LC film. For systematic comparisons, this process step was also conducted with the reference samples (without $\boldsymbol{n}$-BPS treatment). Following UV exposure, the exposed substrates were rinsed (iso-propyl alcohol), dried, and E7 droplets were again deposited on these samples. Now, the contact angle of E7 was measured one more time. Several substrates were investigated for each type of sample and the measured values were averaged.

\section{Preparation of milled particle dispersions}

Dispersions (in heptane) of iron doped lithium niobate particles were fabricated in a planetary ball mill (Retsch PM 200). First, pieces of an Fe: $\mathrm{LiNbO}_{3}$ crystal were manually crushed using an agate mortar and pestle until a powder was obtained. This powder (500 mg) was transferred to a $\mathrm{ZrO}_{2}$ grinding jar and 7-BPS (300 mg) or 11-BPS (300 mg) was added, selectively. Subsequently, each grinding jar was partially filled (1/3rd of the jar height) with small $(1.5 \mathrm{~mm}) \mathrm{ZrO}_{2}$ balls. These balls were preconditioned; they were previously milled in the presence of added heptane and the added heptane was regularly exchanged, and the balls were washed with fresh heptane. During the first repetitions, the fluid phase in the grinding jars had become turbid and was contaminated with abrasion of the balls. The milling process (the preconditioning) was continued until the heptane remained clear. The balls and the milling jars were then washed with filtered heptane. Once the preconditioned balls were added to the grinding jars, heptane $(5 \mathrm{~mL})$ was added through a syringe filter (PTFE $0.2 \mu \mathrm{m})$. The filter was used to avoid the presence of dust in the solvent. The grinding jars were placed in the planetary ball mill, fixed and the milling was conducted at $100 \mathrm{rpm}$. The grinding process was divided in 15 minute intervals, to avoid damage of the mill and continued for $\approx 20 \mathrm{~h}$. Samples were taken during the milling process and stored in Eppendorf tubes.

Dynamic light scattering. Dispersions of milled Fe: $\mathrm{LiNbO}_{3}$ particles (various milling times) were investigated with dynamic light scattering (DLS). The milled dispersions were transferred to light scattering cuvettes and investigated with dynamic light scattering (Malvern Zetasizer).

\section{Conclusions}

Homologues of $n$-BPS with $n=4-12$ were investigated. The yield of the Williamson ether synthesis step was higher for intermediate spacer length (65\%) and smaller for both small and larger spacer length. The yield of the hydrosilation step (76\%) was relatively high and independent of the spacer length. Spin casting from solution followed by thermal annealing and rinsing was found to result in monolayer formation in all homologues as seen in the ellipsometric investigations of surface functionalized Si wafers.

The monolayer thickness was found to depend on both the spacer length and the concentration of the solution used in the spin casting step. These results were instructive to learn about the typical thickness of the obtained monolayers. The use of diluted solutions of species with a short aliphatic spacer group will lead to small monolayer thickness $(d<0.7 \mathrm{~nm})$. One can speculate whether the formation (at least during the annealing process on the spin cast substrates) of lyotropic phases in concentrated solutions of higher homologues can control the kinetics of the grafting process and thereby lead to constant monolayer thickness (apparently with a tilted molecular alignment on the surfaces).

The photochemistry of the benzophenone oxo functional group was tested by exposing surface functionalized samples covered with the LC E7 to UV light. These investigations revealed that the surface energy (contact angle of E7) was indeed varied by photo exposure. These results are promising also for liquid crystal related research because the pretilt angle $^{49}$ of the LC director (which is important for the electrooptic performance) on a surface depends on the (now photo controllable) contact angle. Moreover, $n$-BPS functionalized surfaces can potentially be applied straight-forwardly (for example by spin casting of high molecular weight solvents followed by photo exposure, for example through a photo mask) to fabricate surface coatings with controllable wetting properties.

The synthesized species were used as surfactants in the wet ball milling process of dispersions of small inorganic particles. It turned out that higher homologues are capable of yielding dispersions of functionalized particles and they can outperform well-known surfactants already during the milling process. The particle dispersions obtained are highly useful, because their photochemically active surface functionalization can be addressed by photo-exposure in order to incorporate them in polymer films or in general in soft matter samples.

\section{Conflicts of interest}

There are no conflicts to declare.

\section{Acknowledgements}

The authors express their thanks to Prof. Dr Klaus Huber and Ms Mahnaz Doostdar Kejdehi for fruitful discussions on the properties of solutions and their potential role in the grafting process of $\boldsymbol{n}$-BPS homologues. In addition, the authors would also like to express their thanks to Pd. Dr Hans Egold and Ms Karin Stolte for the measurement of the NMR spectra and assistance with the analysis of the recorded spectra. Funding by the German Research Council (DFG LO 1922/4-1) is gratefully acknowledged.

\section{Notes and references}

1 G. K. Raghuraman, R. Dhamodharan, O. Prucker and J. Rühe, A Robust Method for the Immobilization of Polymer Molecules on $\mathrm{SiO}_{2}$ Surfaces, Macromolecules, 2008, 41, 873-878.

2 S. Hattori, K. Asakawa, S. Mikoshiba, H. Nakamura, A. Hieno, Y. Seino, M. Kanno and T. Azuma, Blockcopolymer Self-Assembly Directed by Photochemically Attached 
Polymer Surface Layer, J. Photopolym. Sci. Technol., 2011, 24, 581-585.

3 Y. Kim, A. Bulusu, A. J. Giordano, S. R. Marder, R. Dauskardt and S. Graham, Experimental Study of Interfacial Fracture Toughness in a $\mathrm{SiN}_{x} / \mathrm{PMMA}$ Barrier Film, ACS Appl. Mater. Interfaces, 2012, 4, 6711-6719.

4 K. M. Carroll, A. J. Giordano, D. Wang, V. K. Kodali, J. Scrimgeour, W. P. King, S. R. Marder, E. Riedo and J. E. Curtis, Fabricating Nanoscale Chemical Gradients with ThermoChemical NanoLithography, Langmuir, 2013, 29, 8675-8682.

5 Y. Kado, M. Mitsuishi and T. Miyashita, Fabrication of Three-Dimensional Nanostructures Using Reactive Polymer Nanosheets, Adv. Mater., 2005, 17, 1857-1861.

6 P. Aliuos, A. Sen, U. Reich, W. Dempwolf, A. Warnecke, C. Hadler, T. Lenarz, H. Menzel and G. Reuter, Inhibition of fibroblast adhesion by covalently immobilized protein repellent polymer coatings studied by single cell force spectroscopy, J. Biomed. Mater. Res., Part A, 2014, 102, 117-127.

7 M. Gianneli, P. W. Beines, R. F. Roskamp, K. Koynov, G. Fytas and W. Knoll, Local and Global Dynamics of Transient Polymer Networks and Swollen Gels Anchored on Solid Surfaces, J. Phys. Chem. C, 2007, 111, 13205-13211.

8 C. Dorrer and J. Rühe, Micro to nano: surface size scale and superhydrophobicity, Beilstein J. Nanotechnol., 2011, 2, 327-332.

9 J. D. Jeyaprakash, S. Samuel and J. Ruehe, Microstructured fluorinated nanofilms, PMSE Prepr., 2004, 90, 290-291.

10 M. Muramatsu, M. Iwashita, T. Kitano, T. Toshima, M. H. Somervell, Y. Seino, D. Kawamura, M. Kanno, K. Kobayashi and T. Azuma, Nanopatterning of diblock copolymer directed self-assembly lithography with wet development, J. Micro/ Nanolithogr., MEMS, MOEMS, 2012, 11, 031305.

11 A.-K. Schuler, O. Prucker and J. Rühe, On the Generation of Polyether-Based Coatings through Photoinduced $\mathrm{C}, \mathrm{H}$ Insertion Crosslinking, Macromol. Chem. Phys., 2016, 217, 1457-1466.

12 K. Li, C. K. Pandiyarajan, O. Prucker and J. Rühe, On the Lubrication Mechanism of Surfaces Covered with SurfaceAttached Hydrogels, Macromol. Chem. Phys., 2016, 217, 526-536.

13 O. Prucker, C. A. Naumann, J. Rühe, W. Knoll and C. W. Frank, Photochemical Attachment of Polymer Films to Solid Surfaces via Monolayers of Benzophenone Derivatives, J. Am. Chem. Soc., 1999, 121, 8766-8770.

14 Y. Shiraishi and T. Hirai, Photochemical desulfurization and denitrogenation of light oil by using benzophenonemodified silica gel as a heterogeneous triplet photosensitizer, Solvent Extr. Res. Dev., Jpn., 2003, 10, 79-85.

15 F. Hoffmann, T. Wolff, S. Minko and M. Stamm, Photochemical structuring and fixing of structures in binary polymer brush layers via $2 \pi+2 \pi$ photodimerization, J. Colloid Interface Sci., 2005, 282, 349-358.

16 K. A. Ibrahim, A. H. Al-Muhtaseb, O. Prucker and J. Rühe, Preparation of hydrophilic polymeric nanolayers attached to solid surfaces via photochemical and ATRP techniques, J. Polym. Res., 2013, 20, 124.
17 A. Hieno, S. Hattori, H. Nakamura, K. Asakawa, Y. Seino, M. Kanno and T. Azuma, Quick Formation of DSA Neutralization Polymer Layer Attached by Reactive Self-Assembled Monolayer, J. Photopolym. Sci. Technol., 2012, 25, 73-76.

18 R. Toomey and Juergen. Ruehe, Surface-attached polymer networks: synthesis and swelling behavior, PMSE Prepr., 2003, 89, 157-158.

19 S. Lenz, S. K. Nett, M. Memesa, R. F. Roskamp, A. Timmann, S. V. Roth, R. Berger and J. S. Gutmann, Thermal Response of Surface Grafted Two-Dimensional Polystyrene (PS)/Polyvinylmethylether (PVME) Blend Films, Macromolecules, 2010, 43, 1108-1116.

20 J. Jarvholm, M. Srinivasarao and L. M. Tolbert, Traversing the "Top-Down/Bottom-Up" Divide: Molecular-Scale Lithography of Self-Assembled Ribbons, J. Am. Chem. Soc., 2009, 131, 398-399.

21 A. Alkan, C. Steinmetz, K. Landfester and F. R. Wurm, Triple-Stimuli-Responsive Ferrocene-Containing PEGs in Water and on the Surface, ACS Appl. Mater. Interfaces, 2015, 7, 26137-26144.

22 S. V. Orski, S. Kundu, R. Gross and K. L. Beers, Design and Implementation of Two-Dimensional Polymer Adsorption Models: Evaluating the Stability of Candida antarctica Lipase B/Solid-Support Interfaces by QCM-D, Biomacromolecules, 2013, 14, 377-386.

23 L. Y. Hwang, H. Götz, C. J. Hawker and C. W. Frank, Glycoacrylate copolymers for bilayer tethering on benzophenonemodified substrates, Colloids Surf., B, 2007, 54, 127-135.

24 W. W. Shen, S. G. Boxer, W. Knoll and C. W. Frank, PolymerSupported Lipid Bilayers on Benzophenone-Modified Substrates, Biomacromolecules, 2001, 2, 70-79.

25 L. Y. Hwang, H. Götz, W. Knoll, C. J. Hawker and C. W. Frank, Preparation and Characterization of GlycoacrylateBased Polymer-Tethered Lipid Bilayers on BenzophenoneModified Substrates, Langmuir, 2008, 24, 14088-14098.

26 S. Loschonsky, K. Shroff, A. Wörz, O. Prucker, J. Rühe and M. Biesalski, Surface-Attached PDMAA-GRGDSP Hybrid Polymer Monolayers that Promote the Adhesion of Living Cells, Biomacromolecules, 2008, 9, 543-552.

27 W. W. Shen, W. Knoll and C. W. Frank, Toward biomimetic lipid bilayers on solid substrates: design and characterization of lipopolymer support, Polym. Mater.: Sci. Eng., 2001, 84, 400-401.

28 A. Woerz, B.-J. Chang, O. Prucker, M. Biesalski and J. Ruehe, Ultrathin polymer layers for the control of cell adhesion - from biocompatible surfaces to living cell chips, Polym. Prepr. (Am. Chem. Soc., Div. Polym. Chem.), 2005, 46, 114-115.

29 L. Braun, S. L. Schafforz and A. Lorenz, Surface grafted crosslinker in polymer network liquid crystals, J. Mol. Liq., 2018, 267, 109-114.

30 L. Braun, Z. Gong, A. Habibpourmoghadam, S. L. Schafforz, L. Wolfram and A. Lorenz, in Emerging Liquid Crystal Technologies XIII, International Society for Optics and Photonics, 2018, vol. 10555, p. 105550 N.

31 W. A. Green, Industrial photoinitiators: a technical guide, CRC Press, Boca Raton, 2010. 
32 Radiation curing: coatings and printing inks; technical basics, applications and trouble shooting, ed. P. Glöckner, T. Jung, S. Struck and K. Studer, Vincentz Network, Hannover, 2008.

33 Radiation curing in polymer science and technology: photoinitiating systems, ed. J. P. Fouassier and J. F. Rabek, Elsevier Applied Science, London, 1993, vol. 2.

34 N. D. Carbone, M. Ene, J. R. Lancaster and J. T. Koberstein, Kinetics and Mechanisms of Radical-Based Branching/ Cross-Linking Reactions in Preformed Polymers Induced by Benzophenone and Bis-Benzophenone Photoinitiators, Macromolecules, 2013, 46, 5434-5444.

35 R. Toomey, D. Freidank and J. Rühe, Swelling Behavior of Thin, Surface-Attached Polymer Networks, Macromolecules, 2004, 37, 882-887.

36 G. Morgese and E. M. Benetti, Polyoxazoline biointerfaces by surface grafting, Eur. Polym. J., 2017, 88, 470-485.

37 A. Lorenz, L. Braun, V. Kolosova, R. Hyman and T. D. Wilkinson, Continuous phase modulation in polymer-stabilized liquid crystals, in Emerging Liquid Crystal Technologies XI, ed. L.-C. Chien, D. J. Broer, H. Kikuchi and N. V. Tabiryan, Proc. SPIE 9769, 2016, p. 976912.

38 S. A. Basun, G. Cook, V. Yu. Reshetnyak, A. V. Glushchenko and D. R. Evans, Dipole moment and spontaneous polarization of ferroelectric nanoparticles in a nonpolar fluid suspension, Phys. Rev. B: Condens. Matter Mater. Phys., 2011, 84, 024105.

39 A. Lorenz, N. Zimmermann, S. Kumar, D. R. Evans, G. Cook, M. Fernández Martínez and H.-S. Kitzerow, Doping a Mixture of Two Smectogenic Liquid Crystals with Barium Titanate Nanoparticles, J. Phys. Chem. B, 2013, 117, 937-941.

40 A. Lorenz, N. Zimmermann, S. Kumar, D. R. Evans, G. Cook and H.-S. Kitzerow, Doping the nematic liquid crystal 5CB with milled $\mathrm{BaTiO}_{3}$ nanoparticles, Phys. Rev. E, 2012, 86, 051704.

41 G. Cook, J. L. Barnes, S. A. Basun, D. R. Evans, R. F. Ziolo, A. Ponce, V. Yu. Reshetnyak, A. Glushchenko and P. P. Banerjee, Harvesting single ferroelectric domain stressed nanoparticles for optical and ferroic applications, J. Appl. Phys., 2010, 108, 064309.

42 I. U. Idehenre, Y. A. Barnakov, S. A. Basun and D. R. Evans, Spectroscopic studies of the effects of mechanochemical synthesis on $\mathrm{BaTiO}_{3}$ nanocolloids prepared using highenergy ball-milling, J. Appl. Phys., 2018, 124, 165501.

43 Y. A. Barnakov, I. U. Idehenre, S. A. Basun, T. A. Tyson and D. R. Evans, Uncovering the mystery of ferroelectricity in zero dimensional nanoparticles, Nanoscale Adv., 2019, 1, 664-670.

44 A. Lorenz, N. Zimmermann, S. Kumar, D. R. Evans, G. Cook, M. F. Martínez and H.-S. Kitzerow, X-ray scattering of nematic liquid crystal nanodispersion with negative dielectric anisotropy [Invited], Appl. Opt., 2013, 52, E1-E5.

45 M. D. Hanwell, D. E. Curtis, D. C. Lonie, T. Vandermeersch, E. Zurek and G. R. Hutchison, Avogadro: an advanced semantic chemical editor, visualization, and analysis platform, J. Cheminf., 2012, 4, 17.

46 M. Ullah, E. Ali and S. B. A. Hamid, Surfactant-Assisted Ball Milling: A Novel Route to Novel Materials with Controlled Nanostructure - A Review, Rev. Adv. Mater. Sci., 2014, 37, 1-14.

47 C. Dhand, N. Dwivedi, X. Jun Loh, A. N. J. Ying, N. Kumar Verma, R. W. Beuerman, R. Lakshminarayanan and S. Ramakrishna, Methods and strategies for the synthesis of diverse nanoparticles and their applications: a comprehensive overview, RSC Adv., 2015, 5, 105003-105037.

48 C. M. Herzinger, B. Johs, W. A. McGahan, J. A. Woollam and W. Paulson, Ellipsometric determination of optical constants for silicon and thermally grown silicon dioxide via a multisample, multi-wavelength, multi-angle investigation, J. Appl. Phys., 1998, 83, 3323-3336.

49 B.-Y. Liu, C.-H. Meng and L.-J. Chen, Role of Monomer Alkyl Chain Length in Pretilt Angle Control of Polymer-Stabilized Liquid Crystal Alignment System, J. Phys. Chem. C, 2017, 121, 21037-21044. 